\title{
A Rare Cause of Pedunculated Polyps Caused by Strongyloides
}

\author{
Omer Yousaf, Arisha Carreon, Ibrahim Mohsin \\ Norton Community Hospital, Ballad Health, Norton, VA, USA
}

Received: 17/10/2021

Accepted: $19 / 10 / 2021$

Published: 03/03/2022

How to cite this article: Yousaf O, Carreon A, Mohsin I. A rare cause of pedunculated polyps caused by Strongyloides. EJCRIM 2022;9: doi:10.12890/2022_003008.

Conflicts of Interests: The authors declare there are no competing interests.

This article is licensed under a Commons Attribution Non-Commercial 4.0 License

\section{ABSTRACT}

Introduction: Strongyloidiasis is a systemic disease caused by the nematode Strongyloides stercoralis. Strongyloides is unique among parasites in that it can remain undetected for decades. Although patients may present with the typical symptoms of a skin rash, cough or diarrhoea, a rare manifestation of infection is pseudopolyposis in the colon. This case highlights the unique finding of pseudopolyposis in a patient with strongyloidiasis with negative stool studies.

Case description: We present the case of an 81-year-old man with a delayed diagnosis of strongyloidiasis. The patient initially presented to the clinic for evaluation of productive cough and was treated empirically for bronchitis. He subsequently developed vomiting and diarrhoea with imaging revealing circumferential wall thickening of the ascending colon, consistent with colitis. Although stool tests were negative, the patient was treated with antibiotics, and then underwent a colonoscopy which revealed multiple polyps. Biopsy results confirmed Strongyloides infection. The patient was treated with ivermectin and improved.

Discussion: This case describes the unique finding of colonic pseudopolyposis in a patient infected with Strongyloides in rural southwest Virginia. Strongyloidiasis is often misdiagnosed due to its non-specific gastrointestinal symptoms and the chronic nature of the disease. Although stool studies are routinely negative for parasitic infection, CT scan findings suggestive of colitis should be followed by colonoscopy with biopsy of any abnormal lesion in the right clinical context. Determining the definitive diagnosis in a timely manner is crucial for establishing the specific treatment and for resolution of the disease.

\section{LEARNING POINTS}

- CT scan findings suggestive of colitis should be followed by colonoscopy with biopsy of any abnormal lesion in the right clinical context; stool studies may be negative, as in this case.

- Internists should know the incidence, even if it is low, of certain parasitic infections in their local area.

- Strongyloides infection in the colon can mimic colitis and initiating the wrong treatment can lead to worse outcomes.

\section{KEYWORDS}

Colitis, pseudopolyposis, Strongyloides stercoralis, pedunculated polyps

\section{INTRODUCTION}

Strongyloides stercoralis is a soil-transmitted nematode which penetrates the skin and is endemic in the tropics and Africa ${ }^{[1]}$. Cases of Strongyloides infection have also occurred in the southeastern United States and southern Europe, although most known cases have been documented in military veterans and immigrants from endemic regions ${ }^{[1]}$. Acute symptoms are usually mistaken for dermatitis. The most feared complication of Strongyloides infection is hyperinfection syndrome and dissemination, which is often associated with human T-lymphotropic virus type ${ }^{[2]}$. Disseminated Strongyloides infection has a mortality rate of $50-87 \%$ even with treatment ${ }^{[3]}$. 
Chronic strongyloidiasis is usually asymptomatic but most often presents with peptic ulcer disease-like symptoms including vague abdominal pain and diarrhoea. We present a unique case of strongyloidiasis manifesting as pedunculated colon polyps in an 81-year-old immunocompetent patient.

\section{CASE DESCRIPTION}

An 81-year-old man initially presented to an urgent care clinic with a productive cough and was empirically treated with azithromycin for bronchitis. Several days later he developed fever and persistent nausea, vomiting and watery diarrhoea, and was admitted to hospital for further evaluation and treatment. His medical history was significant for monoclonal gammopathy of undetermined significance (MGUS), and controlled type 2 diabetes. The patient was not taking any immunosuppressive medications at the time of presentation. On physical examination, the only remarkable finding was pallor and mild abdominal pain in all four quadrants on palpation.

Routine laboratory tests were normal except for mild leucocytosis of 12.5 and peripheral eosinophilia of 15.6\%. A CT scan of the abdomen and pelvis showed a short segment of circumferential wall thickening of the descending colon suggestive of colitis. Blood cultures were negative. Stool studies for ova, larvae, Shiga toxin, Campylobacter, Cytomegalovirus, Giardia and Cryptosporidium were all negative. The patient was started on empiric treatment for infectious colitis and was given levofloxacin and metronidazole. On hospital day 2, a colonoscopy was performed, which revealed multiple non-specific pedunculated polyps in the ascending and transverse colon (Fig. 1). Biopsies were taken which showed inflammatory polyps containing Strongyloides organisms (Fig. 2). The patient was subsequently treated with a four-dose regimen of ivermectin, and a repeat screening colonoscopy 3 months later showed diverticular tics in the descending and transverse colon with no ulcerations or polyps visualized.

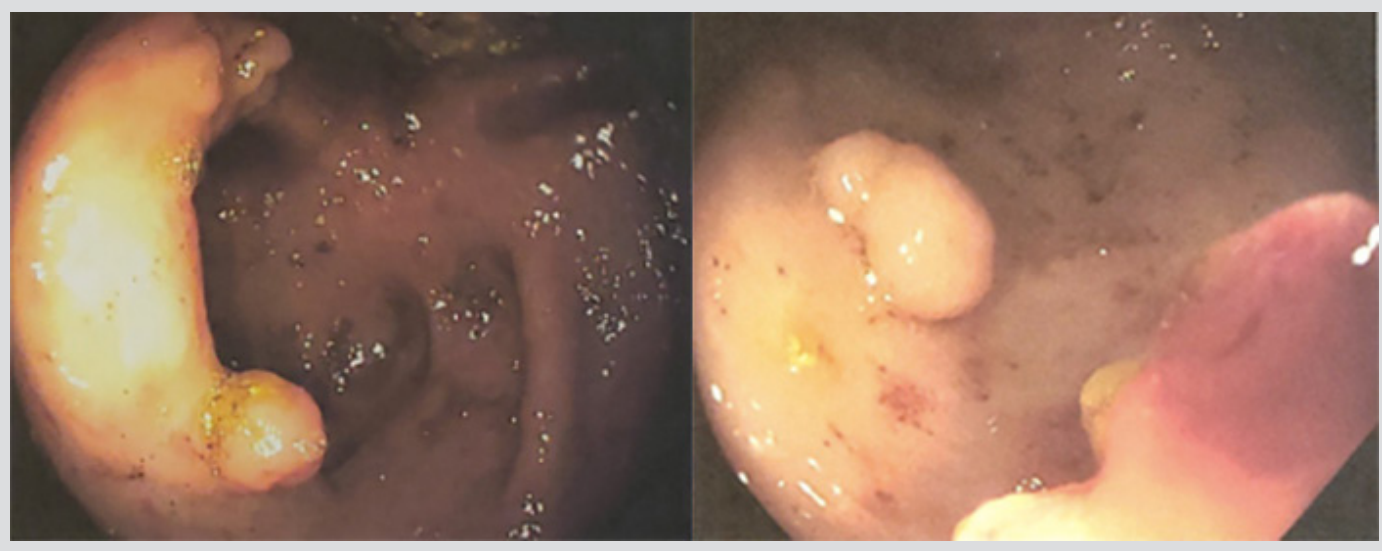

Figure 1. Images taken from colonoscopy which show pedunculated polyps in the transverse colon

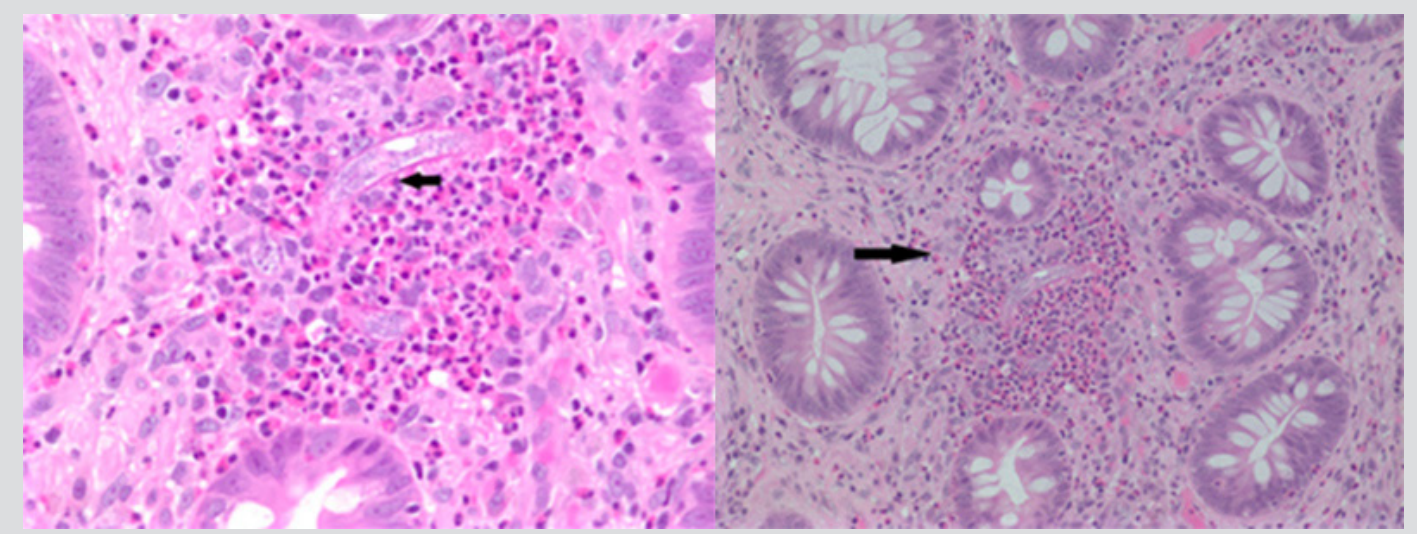

Figure 2. Pathology slides of the lamina propria of the colon mucosa. Black arrows point to the filariform larvae within eosinophilic aggregates

\section{DISCUSSION}

S. stercoralis is a parasitic nematode endemic in tropical countries, although cases have been known to occur in Appalachia and the southeastern United States ${ }^{[1]}$. It penetrates human skin after exposure to contaminated soil. The primary risk factors for infection include exposure to contaminated soil, poor sanitary conditions, and poor personal hygiene. Strongyloides is able to complete its entire lifecycle in a 
human host, leading to chronic infection that can be misdiagnosed at later stages. Initial symptoms include dermatitis, cough and wheezing. Notably, our patient had initially been treated for bronchitis, which may have been a manifestation of early Strongyloides infection. The condition is particularly dangerous in immunocompromised patients in whom disseminated strongyloidiasis with sepsis has a high mortality rate. Peripheral eosinophilia often indicates an underlying helminthic infection, with S. stercoralis infection being the most common aetiology of diagnosis ${ }^{[4]}$. Definitive diagnosis can be made with stool microscopy, however it has a sensitivity of $<50 \%$. Stool nucleic amplification tests have greater specificity than direct stool microscopy, with one systematic review of PCR tests showing a sensitivity of $72 \%$ and specificity of $93 \%{ }^{[5]}$. In our case, routine stool microscopy results were negative for the infection.

This case describes the unique finding of pedunculated polyps on colonoscopy in a patient infected with Strongyloides. Rare biopsy findings in patients with strongyloidiasis may typically include mucosal erythema, ulceration, oedema, erosions and yellowish-white nodules ${ }^{[6,7]}$. These pseudopolyps consist of granulation tissue with an eosinophilic infiltration triggered by the infecting organism. Pseudopolyps containing S. stercoralis have previously been reported in Japan and Korea ${ }^{[7-9]}$. Strongyloides infection in the colon can also mimic ulcerative colitis ${ }^{[10]}$ Diagnosing a patient with ulcerative colitis who actually has Strongyloides infection will most likely result in initiation of steroid therapy. Initiation of corticosteroid therapy can lead a patient to be immunocompromised and can cause a hyperinfection syndrome ${ }^{[11]}$.

To our knowledge, this is a rare reported case in which a patient infected with Strongyloides presented with pedunculated polyps on colonoscopy. For patients with risk factors for strongyloidiasis, negative stool studies with evidence of colitis on imaging and peripheral eosinophilia should prompt colonoscopy evaluation and biopsy in order to expedite a definitive diagnosis and initiate the correct curative treatment. S. stercoralis infection in the colon can mimic colitis and initiating the wrong treatment can lead to worse outcomes.

\section{REFERENCES}

1. Montes M, Sawhney C, Barros N. Strongyloides stercoralis: there but not seen. Curr Opin Infect Dis 2010;23(5):500-504

2. Porto MA, Alcântara LM, Leal M, Castro N, Carvalho EM. Atypical clinical presentation of strongyloidiasis in a patient co-infected with human T cell lymphotrophic virus type I. Am J Trop Med Hyg 2005;72(2):124-125.

3. Caumes E, Keystone JS. Acute strongyloidiasis: a rarity. Chronic strongyloidiasis: a time bomb! J Travel Med 2011:18(2):71-72

4. Salvador F, Sulleiro E, Sánchez-Montalvá A, Saugar JM, Rodríguez E, Pahissa A, et al. Usefulness of Strongyloides stercoralis serology in the management of patients with eosinophilia. Am J Trop Med Hyg 2014;90:830-834.

5. Buonfrate D, Requena-Mendez A, Angheben A, Cinquini M, Cruciani M, Fittipaldo A, et al. Accuracy of molecular biology techniques for the diagnosis of Strongyloides stercoralis infection - a systematic review and meta-analysis. PLoS NegI Trop Dis 2018;12(2):e0006229.

6. Thompson BF, Fry LC, Wells CD, OImos M, Lee DH, Lazenby AJ, et al. The spectrum of GI strongyloidiasis: an endoscopic-pathologic study. Gastrointest Endosc 2004;59(7):906910.

7. Minematsu H, Hokama A, Makishi T, Arakaki K, Kinjo F, Fujita J. Colonoscopic findings and pathologic characteristics of Strongyloides colitis: a case series. Digestion 2011;83:210-214.

8. $\mathrm{Ra} \mathrm{H}$, Chung JW, Chung DH, Kim JH, Kim YJ, Kim KO, et al. Strongyloidiasis presenting as yellowish nodules in colonoscopy of an immunocompetent patient. Clin Endosc 2019;52(1):80-82.

9. Nam S, Han M, Kim Y, Kum Y, Suh I, Bae H. Two cases of strongyloidiasis diagnosed by colonoscopic biopsy. Korean J Pathol 2007;41:343-346.

10. Qu Z, Kundu UR, Abadeer RA, Wanger A. Strongyloides colitis is a lethal mimic of ulcerative colitis: the key morphologic differential diagnosis. Hum Pathol 2009;40(4):572577.

11. Keiser PB, Nutman TB. Strongyloides stercoralis in the immunocompromised population. Clin Microbiol Rev 2004;17(1):208-217. 